\title{
High Mortality Among Patients With Severe COVID- 19 and Do-not-intubate Orders Despite Use of Remdesivir and Dexamethasone
}

Nanna Holm ( $\square$ nanna.holm@regionh.dk)

Copenhagen University Hospital - Amager and Hvidovre Simone Bastrup Israelsen

Copenhagen University Hospital - Amager and Hvidovre

Tamara Theresia Lund

Copenhagen University Hospital - Amager and Hvidovre

Klaus Tjelle Kristiansen

Copenhagen University Hospital - Amager and Hvidovre

Rikke Krogh-Madsen

Copenhagen University Hospital - Amager and Hvidovre

Thomas Benfield

Copenhagen University Hospital - Amager and Hvidovre

Gitte Kronborg

Copenhagen University Hospital - Amager and Hvidovre

\section{Research Article}

Keywords: COVID-19, cCPAP, mechanical ventilation, mortality, Do-not-intubate orders

Posted Date: August 17th, 2021

DOl: https://doi.org/10.21203/rs.3.rs-788108/v1

License: (c) (i) This work is licensed under a Creative Commons Attribution 4.0 International License.

Read Full License 
1 High mortality among patients with severe COVID-19 and do-not-intubate orders

2 despite use of remdesivir and dexamethasone

4 Nanna Holm ${ }^{1}$, Simone Bastrup Israelsen ${ }^{1,2,3}$, Tamara Theresia Lund ${ }^{1}$, Klaus Tjelle Kristiansen ${ }^{4}$, Rikke

5 Krogh-Madsen ${ }^{1,5}$, Thomas Benfield ${ }^{1,2,3}$, Gitte Kronborg ${ }^{1,2}$

$6{ }^{1}$ Department of Infectious Diseases, Copenhagen University Hospital - Amager and Hvidovre,

7 Hvidovre, Denmark

$8 \quad{ }^{2}$ Faculty of Health and Medical Sciences, University of Copenhagen, Denmark

$9 \quad{ }^{3}$ Center for Research and Disruption of Infectious Diseases, Copenhagen University Hospital -

10 Amager and Hvidovre, Hvidovre, Denmark

$11{ }^{4}$ Department of Anesthesiology, Copenhagen University Hospital - Amager and Hvidovre,

12 Hvidovre, Denmark

$13{ }^{5}$ Centre for Physical Activity Research, Copenhagen University Hospital - Rigshospitalet, 14 Copenhagen, Denmark

Corresponding author: 
Abstract

\section{Background}

Moderate to severe respiratory distress among patients with coronavirus disease 2019 (COVID-19) is associated with a high mortality. Dexamethasone and remdesivir were introduced in the second wave of COVID-19 in Denmark.

\section{Methods}

This is a retrospective study of patients with COVID-19 and a supplemental oxygen requirement of $\geq 15$ Liters per minute (L/min). The patients were divided in two groups corresponding to the firstand second wave of COVID-19 and analysed regarding need of ventilator support and mortality dependent on orders to Do Intubate (DI) or Do Not Intubate (DNI), respectively.

\section{Results}

The study included 178 patients. The mortality was $24 \%$ for patients with DI orders $(n=115)$ and $81 \%$ for patients with DNI orders $(n=63)$ increasing to $98 \%(n=46)$ for patients with DNI orders and very high flow oxygen requirements ( $\geq 30 \mathrm{~L} / \mathrm{min}$ ). Use of constant continuous positive airway pressure (cCPAP) increased from $71 \%$ in the first wave to $91 \%$ in the second wave $(p<0.001)$ whereas the use of mechanical ventilation (MV) decreased from $54 \%$ to $28 \%(p=0.005)$.

\section{Conclusion}

The mortality was high for patients with DNI orders and respiratory distress with very high levels in supplemental oxygen in both the first and second wave of COVID-19 despite treatment with dexamethasone and remdesivir and improved prognosis for patients with DI orders. Hence careful evaluation on transition to palliative care must be considered for these patients.

\section{Study Registration}

The study was retrospectively registered and approved by the Danish Patient Safety Authority (record no. 31-1521-309) and the Regional Data Protection Center (record no. WZ20017637-202037). 
50 Keywords: COVID-19, cCPAP, mechanical ventilation, mortality, Do-not-intubate orders.

51

52

53

54

55

56

57

58

59

60

61

62

63

64

65

66

67

68

69

70

71

72

73

\section{Background}

A key feature of coronavirus disease 2019 (COVID-19) caused by severe acute respiratory syndrome coronavirus-2 (SARS-CoV-2) is respiratory distress among hospitalized patients (1).

Respiratory distress may be mild and corrected by low-flow supplemental oxygen while moderate distress requires high-flow or non-invasive ventilation e.g. constant, continuous positive airway pressure (CCPAP). Severe respiratory failure requires mechanical ventilation (MV) or extracorporeal membrane oxygenation (ECMO) (1). Medical treatment includes dexamethasone and remdesivir both introduced as standard treatments in the second wave of COVID-19 in Denmark for patients with moderate to severe respiratory distress $(2,3)$ together with prophylactic anticoagulation due to increased risk of thrombotic events (4). Use of tocilizumab was recommended in March of 2021 for individuals with respiratory failure and inflammation based on clinical trial evidence $(5,6)$.

Multiple studies have demonstrated high mortality rates for patients with COVID-19 and moderate to severe respiratory distress, but the majority of studies are performed on patients with Do Intubate (DI) orders in intensive care unit (ICU) settings $(3,7,8)$. Only few smaller studies have described the mortality among patients with Do Not Intubate (DNI) orders and moderate to severe respiratory distress $(9,10)$.

This study examines the use of ventilator support and mortality in the first- and second wave of COVID-19 for patients with a supplemental oxygen requirement of $\geq 15 \mathrm{~L} / \mathrm{min}$. We evaluate the mortality dependent on orders to DI or DNI. 
This is a single center retrospective study of adult patients ( $\geq 18$ years of age) admitted with COVID-19 at the Department of Infectious Diseases, Copenhagen University Hospital - Amager and Hvidovre from $15^{\text {th }}$ of March to $30^{\text {th }}$ of December 2020. All patients were confirmed SARS-CoV-2 positive by reverse-transcriptase polymerase-chain-reaction (RT-PCR) performed on oropharyngeal swab or lower respiratory tract specimen. Acceptable oxygen saturation was defined as $92-96 \%$ for patients with no previous record of lung diseases and $88-92 \%$ for patients with chronic obstructive pulmonary disease (COPD). Only patients who required $\geq 15 \mathrm{~L} / \mathrm{min}$ of supplemental oxygen to meet the defined criteria for acceptable oxygen saturation were included in this study.

The patients were treated with high flow nasal cannula (HFNC) by the use of Optiflow ${ }^{\circledR}$ and/or CCPAP and a proportion of patients who progressed despite these treatments were transferred to the ICU for MV.

The patients were divided in two groups dependent on their time of admission. Dexamethasone and remdesivir were standard treatments from $17^{\text {th }}$ of June 2020 , hence patients admitted before this date were categorized as Group 1 (admission from $15^{\text {th }}$ of March to $16^{\text {th }}$ of June 2020), and patients after this date were categorized as Group $2\left(17^{\text {th }}\right.$ of June to $30^{\text {th }}$ of December 2020$)$. The majority of patients in Group 1 were admitted during the first wave of the COVID-19 pandemic in Denmark and Group 2 correlates to the second wave. Tocilizumab was introduced as standard treatment after this study period and therefore not available for the included patients.

Based on age, comorbidities and daily activity level each patient was assessed for a DI or DNI order at hospital admission. DNI was assigned to patients who were excluded from MV due to an expected poor outcome. For patients with a DI order initiation of MV was indicated by 1) unacceptable blood oxygen saturation despite maximum supplemental oxygen by HFNC and/or CCPAP, 2) if the patient was not suitable for CCPAP e.g. due to intolerance of the mask or exhaustion or 3 ) if a second organ system failed.

The registered outcomes included in-hospital mortality, use of CCPAP and MV. Patients with supplemental oxygen requirement of $\geq 30 \mathrm{~L} / \mathrm{min}$ were analysed as a subgroup. 
101

102

103 104

105 106

107 108 109 110

Data regarding age, sex, comorbidity, treatment code (DI/DNI), need of supplemental oxygen with HFNC, CCPAP and/or MV, medical treatment of COVID-19 and in hospital death/survival were transferred from the electronic healthcare records to the secure online data platform RedCap hosted by the Capital Region of Denmark.

The study population was characterized using descriptive statistics. Categorical variables were reported as frequencies with percentages. Continuous variables were presented as medians with interquartile range (IQR). Comparisons were performed using $\chi^{2}$ - test and Mann-Whitney $U$ test, as appropriate.

\section{Results}

The study included 178 patients with SARS-CoV-2 infection and need of supplemental oxygen of $\geq 15 \mathrm{~L} / \mathrm{min} ; 82$ patients in Group 1 and 96 patients in Group 2. Demographic factors and comorbidities for the two groups are listed in Table 1. The groups were comparable with no significant differences across in all variables (Table 1).

Table 1. Baseline characteristics of patients in Group 1 and 2

\begin{tabular}{|lrrr|}
\hline & Group 1 & Group 2 & p-value \\
\hline Number of patients (n) & 82 & 96 & \\
\hline Age (IQR) & $71(60-79)$ & $69(55-76)$ & $\mathrm{p}=0.1$ \\
\hline Male, $\mathrm{n}(\%)$ & $54(66 \%)$ & $70(73 \%)$ & $\mathrm{p}=0.3$ \\
\hline BMI*, kg/m2, median (IQR) & $28(24-33)$ & $29(25-32)$ & $\mathrm{p}=0.8$ \\
\hline Any comorbidity, n (\%) & $76(93 \%)$ & $82(85 \%)$ & $\mathrm{p}=0.1$ \\
\hline CVD, $\mathrm{n}(\%)$ & $57(70 \%)$ & $64(66 \%)$ & $\mathrm{p}=0.7$ \\
\hline Hypertension, $\mathrm{n}(\%)$ & $43(52 \%)$ & $41(42 \%)$ & $\mathrm{p}=0.2$ \\
\hline Diabetes, $\mathrm{n}(\%)$ & $31(38 \%)$ & $34(35 \%)$ & $\mathrm{p}=0.7$ \\
\hline COPD, $\mathrm{n}(\%)$ & $9(11 \%)$ & $12(12 \%)$ & $\mathrm{p}=0.8$ \\
\hline
\end{tabular}




\begin{tabular}{llll}
\hline Astma, $n(\%)$ & $7(9 \%)$ & $10(10 \%)$ & $p=0.7$
\end{tabular}

116

*Data regarding Body Mass Index (BMI) was only available for 156 patients (88\%). 'Any comorbidity' includes the listed variables and chronic kidney-or liver disease and cancer. $n=$ number, $I Q R=$ interquartile range, $B M I=$ Body Mass Index, $C V D=$ cardiovascular disease. $C O P D=$ chronic obstructive pulmonary disease.

Treatment with dexamethasone and/or remdesivir was given to few patients in Group 1 as part of a clinical trial. In Group 2 all patients but one (99\%) were treated with dexamethasone and 91 patients (95\%) were treated with remdesivir.

\section{Outcome in Group 1 and 2}

Mortality and need of MV in Group 1 and 2 are shown in Figure 1.

In Group 1, 26 patients (57\%) with DI orders were transferred to ICU for MV and no patients with DI orders died at the ward. The in-hospital mortality was 51\%; 14 patients (29\%) with DI orders and 28 patients $(82 \%)$ with DNI orders $(p<0.001)$.

In Group 2, 19 patients (28\%) with DI orders were transferred to ICU for MV. Two patients with DI orders died before intubation was carried out. The in-hospital mortality was 39\%; 14 patients (21\%) with DI orders and 23 patients $(79 \%)$ with DNI orders $(p<0.001)$.

\section{Figure 1. Outcome for patients in Group 1 and 2.}




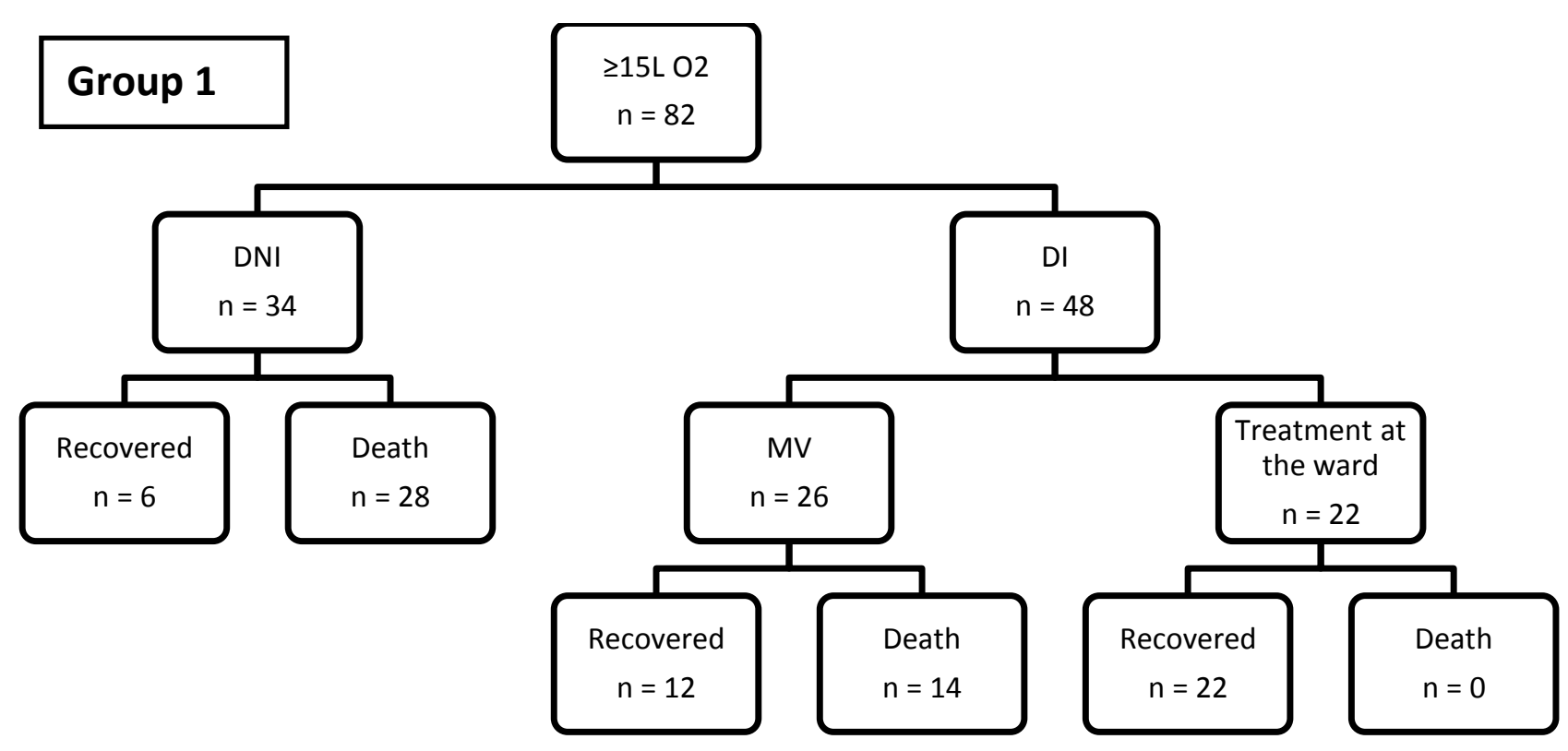

134

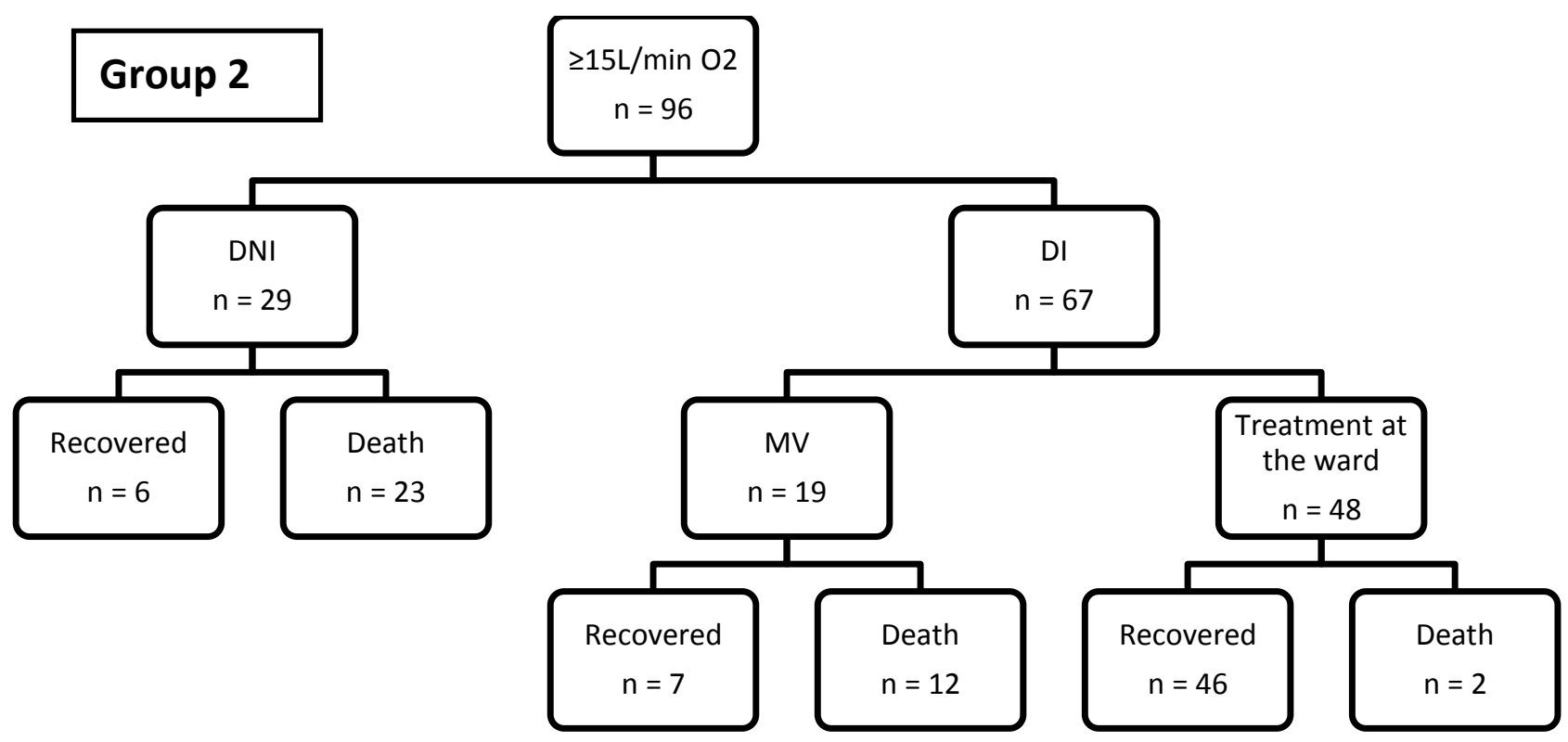

$136 D N I=$ Do Not Intubate,$D I=$ Do Intubate,$M V=$ mechanical ventilation 
139 The use of cCPAP was significantly higher in Group 2, whereas the use of MV was significantly

140 higher in Group 1 (Table 2).

141 The proportion of patients with DNI orders and the overall mortality was higher in Group 1

142 although this was not significant, ( $p=0.1$ and $p=0.09$, respectively). Mortality for patients with DNI 143 orders was comparable between the two groups (Table 2).

144 Table 2. Use of HFNC, cCPAP, MV and mortality rates in Group 1 and 2.

\begin{tabular}{|lcrr|}
\hline & Group 1 & Group 2 & p-value \\
\hline HFNC, all & $81(99 \%)$ & $95(99 \%)$ & \\
\hline & & & \\
\hline cCPAP, all & $58(71 \%)$ & $87(91 \%)$ & $p<0.001$ \\
\hline DI order & $38(79 \%)$ & $63(94 \%)$ & $p=0.02$ \\
\hline DNI order & $20(59 \%)$ & $24(83 \%)$ & $p=0.2$ \\
\hline & & & \\
\hline MV, DI order & $26(54 \%)$ & $19(28 \%)$ & $p=0.005$ \\
\hline & & & \\
\hline In-hospital mortality, all & $42(51 \%)$ & $37(39 \%)$ & $p=0.09$ \\
\hline DI & $14(29 \%)$ & $14(21 \%)$ & $p=0.3$ \\
\hline DNI & $28(82 \%)$ & $23(79 \%)$ & $P=0.8$ \\
\hline
\end{tabular}

HFNC = high flow nasal cannula, $c C P A P=$ constant, continuous positive airway pressure, $M V=$ mechanical ventilation, $D I=$ Do Intubate,$D N I=$ Do Not Intubate .

\section{Outcome for patients with DNI orders}

A comparison of patients with DI- and DNI orders is shown in Table 3. Patients with DNI orders were older, had overall more comorbidities and were more often women. COPD was more frequent in patients with DNI orders whereas patients with DI orders more often were diagnosed with astma.

Table 3. Characteristics and mortality of patients with DNI and DI orders, respectively.

\begin{tabular}{|lrrr|}
\hline & DNI & DI & p-value \\
\hline Number of patients $(n)$ & 63 & 115 & \\
\hline
\end{tabular}




\begin{tabular}{|lrrr|}
\hline Age, median (IQR) & $79(74-83)$ & $64(53-72)$ & $\mathrm{p}<0.001$ \\
\hline Male, n (\%) & $37(59 \%)$ & $87(76 \%)$ & $\mathrm{p}=0.02$ \\
\hline BMI, kg/m2, median (IQR) & $27(23-32)$ & $29(25-32)$ & $\mathrm{p}=0.5$ \\
\hline Any comorbidity, n (\%) & $61(97 \%)$ & $99(86 \%)$ & $\mathrm{p}=0.02$ \\
\hline CVD, n (\%) & $44(70 \%)$ & $77(67 \%)$ & $\mathrm{p}=0.7$ \\
\hline Hypertension, n (\%) & $34(54 \%)$ & $49(43 \%)$ & $\mathrm{p}=0.1$ \\
\hline Diabetes, n (\%) & $28(44 \%)$ & $36(31 \%)$ & $\mathrm{p}=0.08$ \\
\hline COPD, n (\%) & $12(19 \%)$ & $10(9 \%)$ & $\mathrm{p}=0.04$ \\
\hline Astma, n(\%) & $2(3 \%)$ & $15(13 \%)$ & $\mathrm{p}=0.03$ \\
\hline In-hospital mortality, n (\%) & $51(81 \%)$ & $28(24 \%)$ & $\mathrm{p}<0.001$ \\
\hline
\end{tabular}

Any comorbidity includes the listed variables and chronic kidney-or liver disease. IQR = interquartile rage. $B M I=B o d y$ Mass Index,$C V D=$ cardiovascular disease. $C O P D=$ chronic obstructive pulmonary disease.

The in-hospital mortality for patients with DNI orders was $81 \%$. Patients with DNI orders and a supplemental oxygen requirement of $\geq 30 \mathrm{~L} / \mathrm{min}(n=46)$ demonstrated a very high mortality rate of 98\% as 45/46 patients passed away; 25/26 patients in Group 1 and 20/20 patients in Group 2.

\section{Discussion}

Here we show a continued high mortality for patients with DNI orders and severe COVID-19 requiring high-flow supplemental oxygen despite remdesivir, dexamethasone and increased use of cCPAP. However, progression to MV was halved in Group 2 for patients with DI orders.

The very high mortality for patients with DNI orders (81\%) is comparable in Group 1 and 2 and in accordance with two previous reports from the first wave of COVID-19 in Italy of patients with DNI orders and need of non-invasive ventilation (NIV) with mortality rates of $89 \%(n=27)$ and $88 \%$ $(n=25)$, respectively $(9,10)$. In comparison, a study of hospitalized patients with COVID-19 and DNI 
orders not treated with high-flow oxygen (10\% received dexamethasone) found a mortality of $49 \%$ (11).

For individuals with DNI orders requiring very high levels of supplemental oxygen ( $\geq 30 \mathrm{~L} / \mathrm{min})$ the in-hospital mortality proportion was $98 \%$. This leads us to recommend that these patients are carefully evaluated for transition to palliative care in open dialogue with the patient and relatives in order to avoid prolonged struggle and suffering.

The use of CCPAP increased from $71 \%$ in the first wave of COVID-19 to $91 \%$ in the second wave.

This may contribute to the decrease in need of $\mathrm{MV}$ in the second wave together with the introduction of dexamethasone and remdesivir as we have shown in a recent study that included the subset of individuals included here (12). HFNC and cCPAP have been associated with a decrease in use of MV for patients with acute respiratory distress syndrome (ARDS) including patients with COVID-19 (13-17), though the effect still awaits evaluation in randomized controlled trials (18). However, it is still a challenge to identify the patients who would benefit from cCPAP and the patients to whom early intubation should be preferred $(19,20)$.

Though we did not find a significant decrease in overall mortality for patients with severe COVID19 from the first to the second wave this was documented just recently for hospitalized patients with COVID-19 $(12,21)$. However for patients in ICU settings the mortality may be more comparable in the two waves (21-23).

In conclusion the mortality was high for patients with DNI orders and respiratory distress with very high levels in supplemental oxygen in both the first and second wave of COVID-19 despite treatment, while we observed improved prognosis for patients with DI orders. Hence careful evaluation on transition to palliative care must be considered for these patients.

\section{Abbreviations}

COVID-19, coronavirus disease 2019; SARS-CoV-2, severe acute respiratory syndrome coronavirus2; RT-PCR, reverse-transcriptase polymerase-chain-reaction; L/min, Liters per minute; MV, mechanical ventilation; ECMO, extracorporeal membrane oxygenation; ICU, intensive care unit; 
CCPAP, constant, continuous positive airway pressure; HFNC, high flow nasal cannula; NIV, noninvasive ventilation; DI, Do intubate; DNI, Do Not Intubate; IQR, interquartile rage; BMI, Body Mass Index; CVD, cardiovascular disease; COPD, chronic obstructive pulmonary disease; ARDS, acute respiratory distress syndrome.

\section{Declarations}

\section{Ethics approval and consent to participate}

The study was approved by the Danish Patient Safety Authority (record no. 31-1521-309) and the Regional Data Protection Center (record no. WZ20017637-2020-37).

\section{Consent for publication}

Not applicable.

\section{Availability of data and materials}

The data that support the findings of this study are available on reasonable request from the corresponding author, $\mathrm{NH}$. The data are not publicly available as they contain information that could compromise the privacy of research participants.

\section{Competing interests}

The authors declare to have no competing interests.

\section{Funding}

No funding was received. 


\section{Authors' contributions}

$\mathrm{NH}, \mathrm{SI}$ and TL collected data for the study. $\mathrm{NH}$ carried out data-analysis and wrote the manuscript. GK, RM and TB supervised the work on data analysis and manuscript writing and KK reviewed and contributed to the manuscript writing. All authors have read and approved the final manuscript.

\section{Acknowledgement}

Not applicable.

\section{References}

1. Alhazzani W, Moller MH, Arabi YM, Loeb M, Gong MN, Fan E, et al. Surviving Sepsis Campaign: guidelines on the management of critically ill adults with Coronavirus Disease 2019 (COVID-19). Intensive Care Med. 2020;46(5):854-87.

2. Group RC, Horby P, Lim WS, Emberson JR, Mafham M, Bell JL, et al. Dexamethasone in Hospitalized Patients with Covid-19. N Engl J Med. 2021;384(8):693-704.

3. Wang Y, Lu X, Li Y, Chen H, Chen T, Su N, et al. Clinical Course and Outcomes of 344 Intensive Care Patients with COVID-19. Am J Respir Crit Care Med. 2020;201(11):1430-4.

4. Klok FA, Kruip M, van der Meer NJM, Arbous MS, Gommers D, Kant KM, et al. Incidence of thrombotic complications in critically ill ICU patients with COVID-19. Thromb Res. 2020;191:145-7.

5. Group RC. Tocilizumab in patients admitted to hospital with COVID-19 (RECOVERY): a randomised, controlled, open-label, platform trial. Lancet. 2021;397(10285):1637-45.

6. Investigators R-C, Gordon AC, Mouncey PR, Al-Beidh F, Rowan KM, Nichol AD, et al. Interleukin-6 Receptor Antagonists in Critically III Patients with Covid-19. N Engl J Med. 2021;384(16):1491502.

7. Haase N, Plovsing R, Christensen S, Poulsen LM, Brochner AC, Rasmussen BS, et al. Characteristics, interventions and longer-term outcomes of COVID-19 ICU patients in Denmark - a nationwide, observational study. Acta Anaesthesiol Scand. 2020.

8. Richardson S, Hirsch JS, Narasimhan M, Crawford JM, McGinn T, Davidson KW, et al. Presenting Characteristics, Comorbidities, and Outcomes Among 5700 Patients Hospitalized With COVID-19 in the New York City Area. JAMA. 2020;323(20):2052-9.

9. Di Domenico SL, Coen D, Bergamaschi M, Albertini V, Ghezzi L, Cazzaniga MM, et al. Clinical characteristics and respiratory support of 310 COVID-19 patients, diagnosed at the emergency room: a single-center retrospective study. Intern Emerg Med. 2020.

10. Faraone A, Beltrame C, Crociani A, Carrai P, Lovicu E, Filetti S, et al. Effectiveness and safety of noninvasive positive pressure ventilation in the treatment of COVID-19-associated acute hypoxemic respiratory failure: a single center, non-ICU setting experience. Intern Emerg Med. 2020. 
11. van der Veer T, van der Sar-van der Brugge S, Paats MS, van Nood E, de Backer IC, Aerts J, et al. Do-not-intubate status and COVID-19 mortality in patients admitted to Dutch non-ICU wards. Eur J Clin Microbiol Infect Dis. 2021.

12. Benfield T, Bodilsen J, Brieghel C, Harboe ZB, Helleberg M, Holm C, et al. Improved survival among hospitalized patients with COVID-19 treated with remdesivir and dexamethasone. A nationwide population-based cohort study. Clin Infect Dis. 2021.

13. Patel M, Gangemi A, Marron R, Chowdhury J, Yousef I, Zheng M, et al. Retrospective analysis of high flow nasal therapy in COVID-19-related moderate-to-severe hypoxaemic respiratory failure. BMJ Open Respir Res. 2020;7(1).

14. Ferreyro BL, Angriman F, Munshi L, Del Sorbo L, Ferguson ND, Rochwerg B, et al. Association of Noninvasive Oxygenation Strategies With All-Cause Mortality in Adults With Acute Hypoxemic Respiratory Failure: A Systematic Review and Meta-analysis. JAMA. 2020.

15. Ashish A, Unsworth A, Martindale J, Sundar R, Kavuri K, Sedda L, et al. CPAP management of COVID-19 respiratory failure: a first quantitative analysis from an inpatient service evaluation. BMJ Open Respir Res. 2020;7(1).

16. Brusasco C, Corradi F, Di Domenico A, Raggi F, Timossi G, Santori G, et al. Continuous positive airway pressure in COVID-19 patients with moderate-to-severe respiratory failure. Eur Respir J. 2021;57(2).

17. Bellani G, Grasselli G, Cecconi M, Antolini L, Borelli M, De Giacomi F, et al. Noninvasive Ventilatory Support of COVID-19 Patients Outside the Intensive Care Units (WARd-COVID). Ann Am Thorac Soc. 2021.

18. Perkins GD, Couper K, Connolly B, Baillie JK, Bradley JM, Dark P, et al. RECOVERY- Respiratory Support: Respiratory Strategies for patients with suspected or proven COVID-19 respiratory failure;

Continuous Positive Airway Pressure, High-flow Nasal Oxygen, and standard care: A structured summary of a study protocol for a randomised controlled trial. Trials. 2020;21(1):687.

19. Arina P, Baso B, Moro V, Patel H, Ambler G, Group UCLCCC-R. Discriminating between CPAP success and failure in COVID-19 patients with severe respiratory failure. Intensive Care Med. 2021;47(2):237-9.

20. Cabrini L, Ghislanzoni L, Severgnini P, Landoni G, Baiardo Redaelli M, Franchi F, et al. Early versus late tracheal intubation in COVID-19 patients: a pro-con debate also considering heart-lung interactions. Minerva Cardioangiol. 2020.

21. Dutch C, Thrombosis C, Kaptein FHJ, Stals MAM, Grootenboers M, Braken SJE, et al. Incidence of thrombotic complications and overall survival in hospitalized patients with COVID-19 in the second and first wave. Thromb Res. 2021;199:143-8.

22. Contou D, Fraisse M, Pajot O, Tirolien JA, Mentec H, Plantefeve G. Comparison between first and second wave among critically ill COVID-19 patients admitted to a French ICU: no prognostic improvement during the second wave? Crit Care. 2021;25(1):3.

23. Karagiannidis C, Windisch W, McAuley DF, Welte T, Busse R. Major differences in ICU admissions during the first and second COVID-19 wave in Germany. Lancet Respir Med. 2021;9(5):e47-e8. 